\title{
Completeness degree
}

\section{A generalization of dimension}

J. M. A arts (Cambridge, Mass.)

All spaces under discussion are metrizable

\section{Introduction.}

1.1. In this paper we give an outline of a generalization of dimension theory by replacing the empty set in the definition of inductive dimension by a topologically complete space. The most important notion is that of strong inductive completeness degree which is analogous to strong inductive dimension Ind. The formal definition is as follows (cf. [7], p. 9).

Definition. A space $X$ has strong inductive completeness degree -1 , Icd $X=-1$, if $X$ is topologically complete. If for any disjoint closed sets $F$ and $G$ of a space $X$ there exists an open set $U$ such that $F \subset U \subset X \backslash G$ and $\operatorname{Icd} B(U) \leqslant n-1$, then $X$ has strong inductive completeness degree $\leqslant n$, $\operatorname{Icd} X \leqslant n$. $\operatorname{Icd} X=n$ if $\operatorname{Icd} X \leqslant n$ and $\operatorname{Icd} X \leqslant n-1$. If $\operatorname{Icd} X \leqslant n$ for each $n$, then $\operatorname{Icd} X=\infty$.

1.2. Dimension and completeness degree are related by the following theorem which justifies the use of the term "completeness degree".

MaIN Theorem. A metric space $X$ has strong inductive completeness degree $\leqslant n$ if and only if $X$ has a topologically complete extension $Y$ suoh that $\mathrm{I} \backslash X$ has strong inductive dimension $\leqslant n$.

In view of this theorem Icd $X \leqslant n$ is an internal, necessary and sufficient condition on a metric space $X$ so that $X$ has a topologically complete extension $Y$ with Ind $Y \backslash X \leqslant n$. This theorem is proved in section 3 . The main theorem can be restated by introducing the notion of completeness deficiency $\left(G_{\delta}\right.$-deficiency in [1]). By the completeness deficiency of a space $X$ we mean the least integer $n$ such that $X$ has a complete extension $Y$ with Ind $Y \backslash X=n$ (of course, we allow $n$ to be $\infty$ ). Then, the main theorem says: the strong inductive] completeness degree of $X$ equals the completeness deficiency of $X$.

1.3. Now, by replacing Ind by Icd several theorems can be obtained from dimension theory. This is done in sections 4 and 5 . 
On the other hand, in view of the main theorem, some "dual theorems" can be expected to hold. See 4.5 and 4.7 .

The device of replacing Ind by Icd does not work in the following cases: the sum theorem in its full generality (there is a restricted sum theorem in 4.6) and the product theorem. See 2.4, 4.6 and 4.8 .

1.4. We will define small inductive completeness degree and covering completeness degree as analogies to small inductive dimension and covering dimension respectively. It turns out that covering completeness degree and strong inductive completeness degree coincide. Small and strong inductive completeness degree are equal for separable spaces (see section 7 and 6 respectively).

1.5. Other generalizations of dimension have been discussed by J. de Groot and T. Nishiura ([2], [3], [4] and [9]).

\section{Preliminaries and examples.}

2.1. The closure operator will be denoted by an upper bar. $B_{X}(U)$ (or $B(U)$ when no confusion is likely to arise) denotes the boundary of $U$ in $X$. The complement of $B$ in $A$ is denoted by $A \backslash B$. For shortness sake, "complete" means "topologically complete". $Y$ is called an extension of $X$ if $X$ is a dense subset of $Y$. For metric spaces the dimension functions $\operatorname{dim}(=$ covering dimension) and Ind (= strong inductive dimension) coincide. We use Ind and dim indiscriminately.

2.2. We frequently use the following consequence of the heriditary normality of a metric space.

LEMMA. For any subsets $A$ and $B$ of a space $X$ satisfying $A \cap \bar{B}=\varnothing$ and $\bar{A} \cap B=\varnothing$ there exist open sets $U$ and $V$ such that $A \subset U, B \subset V$ and $U \cap V=\varnothing$ (cf. [7], p. 3).

2.3. A topological property $P$ will be called hereditary with respect to $G_{\delta}$-subsets if whenever a space $X$ has property $\mathrm{P}$ then every $G_{\delta}$-subset of $X$ has property $P$. In the sequel, many proofs are based upon the following property of complete extensions of a space.

Lencma. Let $\mathrm{P}$ be a topological property hereditary with respect to $G_{\delta}$-subsets. If for a space $X$ there exists a complete extension with the property $\mathrm{P}$, then each complete extension $Y$ of $X$ contains a complete extension of $X$ with the property $\mathrm{P}$.

This lemma can easily be deduced from the following well-known theorems.

THeonem. (Alexandrov-Hausdorff.) $X$ is complete if and only if $X$ is a $G_{\delta}$-subset of each space $Y$ which contains $X$ (see [6], p. 337).

THEOREM. (Lavrientiev.) Each homeomorphism between subspaces $A$ and $B$ of complete spaces $X$ and $Y$ respectively, can be extended to a homeomorphism between $G_{\delta}$-subsets of $X$ and $Y$ (see [6], p. 335).
Proof of the lemma. Let $I_{1}$ be a complete extension of $X$ with property P. Due to the theorem of Lavrientiev, the identity map of $X$ onto itself can be extended to a homeomorphism between $G_{\delta}$-subsets $Z$ and $Y_{2}$ of $Y_{1}$ and $Y$ respectively. Due to the theorem of AlexandrovHausdorff, $Y_{2}$ is complete and this set has property $P$. Indeed, $P$ is a topological property and $Z$ has $\mathrm{P}$ because $\mathrm{P}$ is hereditary with respect to $G_{\delta}$-subsets.

2.4. We now discuss the existence of spaces with strong inductive completeness degree $n$. The main theorem (1.2), which will be proved in section 3 , is used.

THEOREM. Let $X$ be a non-complete space and $Y$ a union of a locally countable family $\left\{C_{a}\right\}_{\alpha \in A}$ of compact subspaces. If $Z$ is a complete extension of $X \times Y$, then $\operatorname{dim} Y \leqslant \operatorname{dim}(Z \backslash X \times Y)$.

Proof. First suppose that $Y$ is compact. Let $\widehat{X}$ be a complete extension of $X$. We assume that $X \times Y$ is embedded in $\widetilde{X} \times Y$ in the natural way. According to lemma 2.3 there exists a complete extension $Y_{1}$ of $X \times Y$, which is contained in $\widetilde{X} \times Y$, such than $\operatorname{dim}\left(Y_{1} \backslash X \times Y\right)$ $\leqslant \operatorname{dim}(Z \backslash X \times Y)$. Let $p$ denote the natural projection of $\underset{\widetilde{X}}{\widetilde{X}} \times Y$ onto $\tilde{X}$. $p$ is closed since $Y$ is compact. Because $Y_{1}$ is complete, $\widetilde{X} \times Y \backslash Y_{1}$ is an $F_{\sigma}$-subset of $\tilde{X} \times Y$. Consequently, $p\left(\tilde{X} \times Y \backslash Y_{1}\right)$ is an $F_{\sigma}$-subset of $\tilde{X}$. $p\left(\tilde{X} \times Y \backslash I_{1}\right)$ is contained in $\tilde{X} \backslash X$, but it is not all of $\bar{X} \backslash X$ since $X$ is not complete. Let $x \in(\widetilde{X} \backslash X) \backslash p\left(\widetilde{X} \times Y \backslash I_{1}\right)$. Then, $p^{-1}(x) \subset Y_{I} \backslash X \times Y$. Since $p^{-1}(x)$ is homeomorphic with $Y, \operatorname{dim} p^{-1}(x)=\operatorname{dim} Y$. Consequently, $\operatorname{dim} Y \leqslant \operatorname{dim}\left(Y_{1} \backslash X \times Y\right)$.

If $Y$ is not compact, then due to the sum theorem of dimension theory ([7], p. 17) for some $\alpha \in A$ we have $\operatorname{dim} C_{\alpha}=\operatorname{dim} Y$. Now, the same argument as above can be used to show that for each complete extension $Z$ of $X \times Y$ there is a topological copy of $C_{\alpha}$ in $Z \backslash X \times Y$. Hence

$$
\operatorname{dim}(Z \backslash X \times Y) \geqslant \operatorname{dim} C_{a}=\operatorname{dim} T \text {. }
$$

EXAMPLE. Let $Q, I$ and $R$ denote the rationals, the unit interval and the real numbers, respectively (endowed with the usual topology). Then $\operatorname{Icd} Q \times I^{n}=\operatorname{Icd} Q \times R^{n}=n$.

Indeed, from the theorem above, it follows that for each complete extension $Z$ of $Q \times I^{n}$ we have $\operatorname{dim}\left(Z \backslash Q \times I^{n}\right) \geqslant \operatorname{dim} I^{n}=n$. On the other hand, $I^{n+1}$ can be regarded as a complete extension of $Q \times I^{n}$ such that $\operatorname{dim}\left(I^{n+1} \backslash Q \times I^{n}\right)=n$. It follows that the completeness deficiency of $Q \times I^{n}$ is $n$. By the main theorem $\operatorname{Icd} Q \times I^{n}=n$. Similarly, $\operatorname{Ied} Q \times R^{n}=n$ is proved.

Proposition. For each $k(-1 \leqslant k \leqslant n-1)$ there is a subset $X$ of the $n$-dimensional Euclidean space $\varepsilon^{n}$ with $\operatorname{Icd} X=k . \varepsilon^{n}$ contains no sets of higher Ied. 
Proof. In view of the example above it suffices to show that if $X \subset \varepsilon^{n}$, then $\operatorname{Icd} X \leqslant n-1$. Obviously, $\vec{X}$ is complete. Ind $(\bar{X} \backslash X) \leqslant n-1$ in view of [7], p. 98. By the main theorem it follows that Ied $X \leqslant n-1$.

2.5. EXAMPLE. Recall that a space $X$ is called totally imperfect if it contains no (homeomorphic) copy of the Cantor set. Let $X$ be a separable complete space of dimension $n \geqslant 2$. We will exhibit a subset $Y$ with $\operatorname{Icd} Y \geqslant[n / 2]-1$ ([n/2] denotes the greatest integer which does not exceed $n / 2)$. By a theorem of Bernstein ([6], p. 422) $X$ can be decomposed into two totally imperfect, mutually disjoint subsets $Y$ and $Z$. Wither $\operatorname{dim} Y \geqslant[n / 2]$ or $\operatorname{dim} Z \geqslant[n / 2]$ (otherwise, by [7], p. 19, $\operatorname{dim} X \leqslant \operatorname{dim} X+$ $+\operatorname{dim} Z+1 \leqslant 2([n / 2]-1)+1<n)$. Assume $\operatorname{dim} Z \geqslant[n / 2]$. Then Icd $Y$ $\geqslant[n / 2]-1$. Indeed, due to lemma 2.3 there exists a complete extension $X_{1}$ of $Y$ such that $Y \subset X_{1} \subset X$ and $\operatorname{dim}\left(X_{1} \backslash Y\right)$ is minimal. $X \backslash X_{1}$ is an $F_{\sigma}$-subset of $X$ since $X_{1}$ is complete (theorem of Alexandrov-Hausdorff; see 2.3.). Then, $X \backslash X_{1}$ is a Borel set (for definition see [6], p. 250). Consequently, if $X \backslash X_{1}$ is uncountable, it contains a copy of the Cantor set ([6], p. 355, theorem of Alexandrov-Hausdorff). However, $X \backslash X_{1} \subset Z$ and $Z$ contains no Cantor sets. It follows that $X \backslash X_{1}$ is countable, whence zero-dimensional. Because $Z=\left(X \backslash X_{1}\right) \cup\left(X_{1} \backslash Y\right), \operatorname{dim}\left(X_{1} \backslash Y\right) \geqslant[n / 2]-1$. From the main theorem it now follows that Icd $Y \geqslant[n / 2]-1$.

3. Proof of the main theorem. We first prove some [lemmas.

3.1. Lemma. If $A$ is a closed subset of $X$, then $\operatorname{Icd} A \leqslant \operatorname{Icd} X$.

Remark. Actually Icd $X \leqslant n$ is an invariant under the taking of $G_{\delta}$-subsets (see 4.4).

Proof. The proof is by induction on $\operatorname{Icd} X$. If $\operatorname{Icd} X=-1$, then $X$ is complete, which implies that $A$ is complete; hence $\operatorname{Ied} A=-1$. Assume the lemma for spaces $X$ with $\operatorname{Icd} X \leqslant n-1$. If $F$ and $G$ are disjoint closed subsets of the subspace $A$ of a space $X$ with $\operatorname{Icd} X \leqslant n$, then there exists an open set $U$ of $X$ such that $F \subset U \subset X \backslash G$ and $\operatorname{Ied} B(U) \leqslant n-1$, because $F$ and $G$ are also closed in $X$. Then, $F \subset U \cap A \subset A \backslash G$ and $B_{A}(U \cap A)$ $C B(U) \cap A$. By the induction hypothesis, $\operatorname{Icd} B_{A}(U \cap A) \leqslant n-1$. Hence
$\operatorname{Icd} A \leqslant n$.

3.2. Lema. Let $A$ be a subset of $X$ with $\operatorname{Ied} A \leqslant n(n \geqslant 0)$. For any disjoint closed subsets $F$ and $G$ of $X$ there exists an open set $U$ such that $F \subset U \subset X \backslash G$ and $\operatorname{Icd}(B(U) \cap A) \leqslant n-1$.

Proof. Since $X$ is normal, there exist open sets $V$ and $W$ for which $F \subset V, G \subset W$ and $\vec{V} \cap \bar{W}=\emptyset$. Because $\operatorname{Ied} A \leqslant n$, there exists an open subset $D$ of $A$ such that $\bar{V} \cap A \subset D \subset A \backslash \bar{W}$ and $\operatorname{Icd} B_{A}(D) \leqslant n-1$. Neither of the disjoint sets $F \cup D$ and $G \cup(A \backslash \bar{D})$ contains a cluster point of the other. By 2.2 there exists an open set $U$ such that $F \cup D \subset U$ and
$\bar{U} \cap(G \cup(A \backslash \bar{D}))=\emptyset . B(U) \subset \bar{U} \cup$ and $B(D) \cap A$ of $B_{A}(D)$. From lemma 3.1 it now follows that $\operatorname{Ied}(B(U) \cap A)$ $\leqslant n-1$.

Remark. If in the lemma "Icd" is replaced by "Ind", then one gets a well-known result from dimension theory, which can be proved similarly by replacing "Icd" by "Ind".

3.3. Lema. The union of a $\sigma$-locally finite collection of $F_{\sigma}$-substes of a space $X$, is an $F_{\sigma}$-subset of $X$.

Proof. For each $i=1,2, \ldots$, let $\left\{F_{\gamma_{i}} \mid \gamma_{i} \in \Gamma_{i}\right\}$ be a locally finite collection of $F_{\sigma}$-subsets. For each index $\gamma_{i}$ let $F_{\gamma_{i}}=\bigcup\left\{F_{\gamma_{i}}^{k} \mid k=1,2, \ldots\right\}$, where each $F_{\gamma_{i}}^{k}$ is closed. Then, for each $k$ and $i$ the set $\bigcup\left\{F_{\gamma_{i}}^{k} \mid \gamma_{i} \in \Gamma_{i}\right\}$ is closed, since $\left\{F_{\gamma_{i}}^{k} \mid \gamma_{i} \in \Gamma_{i}\right\}$ is locally finite. Clearly, $\bigcup\left\{F_{\gamma_{i}} \mid \gamma_{i} \in \Gamma_{i}\right.$; $i=1,2, \ldots\}=\bigcup\left\{F_{\gamma_{i}}^{k} \mid \gamma_{i} \in \Gamma_{i} ; i=1,2, \ldots ; k=1,2, \ldots\right\}$ and the lemma follows.

3.4. Proof of the main theorem 1.2. "if"-part. The proof is by induction on $\operatorname{dim}(X \backslash X)$. The "if"-part obviously holds for $n=-1$. Assume the "if"-part for $X$ and $\bar{Y}$ with $\operatorname{dim}(\bar{X} \backslash X) \leqslant n-1$. Suppose that $Y$ is a complete extension of $X$ with $\operatorname{dim}(Y \backslash X) \leqslant n$. Let $F$ and $G$ be disjoint closed subsets of $X$. Delete $\bar{F} \cap \bar{G}$ from $\bar{Y} . \bar{Y}_{1}=\Pi \backslash(\bar{F} \cap \bar{G})$ is a complete extension of $X$ and $\operatorname{dim}\left(Y_{1} \backslash X\right) \leqslant n$. Then, in the space $Y_{1}$ we have $\bar{F} \cap \bar{G}=\varnothing$. It follows that in $\bar{Y}_{1}$ there exists an open set $U$ such that $\bar{F} \subset U \subset Y_{1} \backslash \bar{G}$ and $\operatorname{dim}\left(B(U) \cap\left(Y_{1} \backslash X\right)\right) \leqslant n-1$ (see remark in 3.2.). By the induction hypothesis $\operatorname{Icd}(B(U) \cap X) \leqslant n-1 . B_{X}(U)$ is a closed subset of $B(U) \cap X$ and by lemma 3.1 we have $\operatorname{Iod} B_{X}(U) \leqslant n-1$. This proves that $\operatorname{Ied} X \leqslant n$.

"only if"-part. The proof is by induction on Ied $X$. Suppose Ied $X$ $=n \geqslant 0$. (The case $n=-1$ is obvious.)

A. First, take an arbutrary complete extension $Y$ of $X$. We will delete an $F_{\sigma}$-subset of $X$ so that the complement of $X$ becomes $\leqslant n$-dimensional.

B. In $Y$ there exists a $\sigma$-locally finite collection of open subsets $\left\{U_{\gamma} \mid \gamma \in \Gamma\right\}$ and a collection of closed subsets $\left\{F_{\gamma} \mid \gamma \in \Gamma\right\}$ such that

1) $\mathbb{F}_{\gamma} \subset U_{\gamma}$, for each $\gamma \in \Gamma$ and

2) if $V_{\gamma}, \gamma \in \Gamma$, is an open subset such that $F_{\gamma} \subset V_{\gamma} \subset U_{\gamma}$, then $\left\{V_{\gamma} \mid \gamma \in T\right\}$ is a $\sigma$-locally finite base.

Indeed, let $w_{i}$ be the family of all open balls of diameter $<1 / i$ (in some metric of $Y$ ). Let $\mathcal{U}_{i}=\left\{U_{a}^{i} \mid \alpha \epsilon A_{i}\right\}$ be a locally finite covering which is a refinement of $w_{i}$. Since $\mho_{i}$ is a locally finite covering, there is a closed covering $\left.\left\{F_{a}^{i}\right\} \alpha \epsilon A_{i}\right\}$ such that $F_{\alpha}^{i} \subset U_{a}^{i}$ for every $\alpha \in A_{i}$ (see e.g. [7], p. 2). As is easily seen $\left\{U_{a}^{i} \mid \alpha \in A_{i} ; i=1,2, \ldots\right\}$ and $\left\{F_{a}^{i} \mid \alpha \in A_{i}\right.$; $i=1,2, \ldots\}$ satisfy all conditions required. 
C. By lemma 3.2 for each $\gamma \in \Gamma$ there exists an open set $V_{\gamma}$ such that $F_{\gamma} \subset V_{\gamma} \subset U_{\gamma}$ and $\operatorname{Ied}\left(B\left(V_{\gamma}\right) \cap X\right) \leqslant n-1$.

D. By the induction hypothesis each set $B\left(V_{\gamma}\right) \cap X$ has a topologically complete extension $C_{\gamma}$ such that $C_{\gamma} \backslash\left(B\left(V_{\gamma}\right) \cap X\right)$ has dimension $\leqslant n-1$. By lemma 2.3 we may assume that $C_{\gamma} \subset B\left(V_{\gamma}\right)$. Since $B\left(V_{\gamma}\right)$ is closed, $B\left(V_{\gamma}\right) \backslash C_{\gamma}$ is an $F_{\sigma}$-subset of $X$.

E. We delete from $Y$ the set $U\left\{B\left(V_{\gamma}\right) \backslash C_{\gamma} \mid \gamma \in \Gamma\right\}$ and obtain a space $Y_{1}$. Due to lemma 3.3 and $B$ the set $\bigcup\left\{B\left(V_{\gamma}\right) \backslash C_{\gamma} \mid \gamma \in T\right\}$ is an $F_{\sigma}$-subset of $Y$. It follows that $Y_{1}$ is complete. We will show that $\operatorname{dim}\left(Y_{1} \backslash X\right) \leqslant n$.

F. From B it follows that $\left\{V_{\gamma} \cap\left(Y_{1} \backslash X\right) \mid \gamma \in \Gamma\right\}$ is a $\sigma$-locally finite base for the subspace $Y_{1} \backslash X$. Now,

$$
B_{Y_{1} \backslash X}\left(V_{\gamma} \cap\left(Y_{1} \backslash X\right)\right) \subset B_{Y_{1}}\left(V_{\gamma}\right) \frown\left(Y_{1} \backslash X\right)
$$

as is easily seen,

and

$$
B_{Y_{1}}\left(V_{\gamma}\right) \cap\left(Y_{1} \backslash X\right) \subset B\left(V_{\gamma}\right) \cap\left(Y_{1} \backslash X\right)
$$

$$
B\left(V_{\gamma}\right) \cap\left(I_{1} \backslash X\right) C C_{\gamma} \backslash\left(B\left(V_{\gamma}\right) \cap X\right) \quad(\text { by } \mathrm{D}) .
$$

It follows that

$$
\operatorname{dim} B_{Y_{1} \backslash X}\left(V_{\gamma} \cap\left(Y_{1} \backslash X\right)\right) \leqslant \operatorname{dim} C_{\gamma} \backslash\left(B\left(V_{\gamma}\right) \cap X\right) \leqslant n-1 \quad \text { (see D). }
$$

By [7], p. 18 we have $\operatorname{dim}\left(Y_{1} \backslash X\right) \leqslant n$.

3.5. Corollary. If $\operatorname{Icd} X=n$ and $\operatorname{dim} X=m$, then $X$ has a complete. extension $Y$ such that $\operatorname{dim} Y=m$ and $\operatorname{dim}(Y \backslash X)=n$.

Remark. This result is in contrast to the situation for compactifications. There is an example of Nishiura [8] of a space $X$ with the following property: if $Y$ is a compactification of $X$ such that $\operatorname{dim}(I \backslash X)$ is minimal, then $\operatorname{dim} Y>\operatorname{dim} X$.

Proof. By the preceding theorem there is a complete space $Y_{1}$ such that $\operatorname{dim}\left(Y_{1} \backslash X\right)=n . X$ is an $m$-dimensional subset of $Y_{1}$. By [7], p. 32, there is an $m$-dimensional (complete) $G_{\delta}$-subset $Y$ of $Y_{1}$ such that $X \subset X \subset Y_{1} \cdot \operatorname{dim}(Y \backslash X)=n$ as follows from $\operatorname{Icd} X=n$ and the preceding theorem.

4. A theory for Ied. In this section we check which of the fundamental theorems of dimension theory can be generalized to theorems for Icd. We also prove some "dual" theorems.

4.1. First, we give two simple propositions which can be proved by induction.

Proposttion. $\operatorname{Icd} X \leqslant \operatorname{dim} X$.
Proposition. If $\operatorname{Icd} X=n$, then for each $k,-1 \leqslant k \leqslant n$, there exists a closed subset $F_{k}$ such that $\operatorname{Icd} F_{k}=k$.

The first proposition follows from the comparison of the definitions of Icd and strong inductive dimension. To prove the second proposition, observe that there exist disjoint elosed sets $F$ and $G$ in $X$ such that for every open set $U$ with $F \subset U \subset X \backslash G$ we have $\operatorname{Ied} B(U) \geqslant n-1$. Indeed, $\operatorname{Icd} X \$ n-1$. Since Icd $X \leqslant n$, for some $U$ with $F \subset U \subset X \mathbb{G} G$ we have $\operatorname{Icd} B(U) \leqslant n-1$. Hence $\operatorname{Icd} B(U)=n-1$ for such a $U$. Define $B(U)$ $=F_{n-1} . \quad F_{n-l}(2 \leqslant l \leqslant n)$ is defined by induction. Obviously, $X=F_{n}$ and. $\varnothing=F_{-1}$ satisfy the conditions of the proposition.

4.2. In order to apply dimension theory in combination with the main theorem, the following two lemmas are used.

LEMra. The union of a locally finite collection of $G_{\delta}$-subsets of a space $X$ is a $G_{\delta}$-subset of $X$.

Proof. Let $\left\{G_{a} \mid a \in A\right\}$ be a locally finite collection of $G_{\delta}$-subsets of $X$. Let $\left\{U_{a} \mid \alpha \in A\right\}$ be a locally finite collection of open sets such that $G_{a} \subset U_{a}$ for each $a\left([5]\right.$, p. 158). Let $G_{a}=\bigcap\left\{D_{a}^{i} \mid i=1,2, \ldots\right\}$, each $U_{a}^{i}$ being an open subset of $X$. We may assume that $U_{a}^{i} \subset U_{a}$ for each $i$ and $\alpha$. Then

$$
\bigcup\left\{G_{\alpha} \mid a \in A\right\}=\bigcap\left\{\bigcup\left\{U_{a}^{i} \mid \alpha \in A\right\} \mid i=1,2, \ldots\right\} .
$$

Indeed, obviously

$$
\bigcup\left\{G_{a} \mid a \in A\right\} \subset \cap\left\{\bigcup\left\{U_{a}^{i} \mid a \in A\right\} \mid i=1,2, \ldots\right\} .
$$

If $x \notin \dot{U}\left\{G_{a} \mid a \in A\right\}$, then there is a neighbourhood $U$ of $x$ which meets at most finitely many $U_{a}: U_{a_{1}}, \ldots, U_{a_{n}}$ say. Since $x \notin \bigcup\left\{G_{a} \mid a \in A\right\}$, it follows that $x \in G_{a_{1}} \cup \ldots \cup G_{a_{n}}$. Then, $i_{1}, \ldots, i_{n}$ can be selected such that $x \notin U_{a_{k}}^{i_{k}}, k=1, \ldots, n$. If $i=\operatorname{maximum}\left\{i_{1}, \ldots, i_{n}\right\}$, then $x \notin \bigcup\left\{U_{a}^{i} \mid \alpha \in A\right\}$.

4.3. Lemma. If $\left\{G_{\alpha} \mid \alpha \in A\right\}$ is a locally finite family of subsets of a subspace $X$ of a space $T$, then there exists an open subset $U$ of $Y$ such that $X \subset U \subset Y$ and $\left\{G_{a} \mid \alpha \in A\right\}$ is a locally finite collection in the subspace $U$,

Proof. For each point $x \in X$, one can select an open neighbourhood $U(x)$ of $x$ in $Y$ such that $U(x) \cap X$ meets at most finitely many elements of $\left\{G_{a} \mid a \in A\right\}$. Then $U=\bigcup\{U(x) \mid x \in X\}$ satisfies all conditions required.

4.4. We now investigate the values of Icd for subsets of a space $X$. As shown by the examples in 2.4 and 2.5 , completeness degree is not a monotone function. However, since a $G_{\delta}$-subset of a complete space $X$ (i.e. $\operatorname{Icd} X=-1$ ) is complete, the following theorem can be expected to hold.

THeOREM. If $A$ is a $G_{\delta}$-subset of $X$, then $\operatorname{Icd} A \leqslant \operatorname{Icd} X$.

Proof. Let $A=\cap\left\{U_{i} \mid i=1,2, \ldots\right\}$, each $U_{i}$ being open in $X$. Due to the main theorem there exists a complete extension of $X$ for which Fundamenta Mathematicae, $T$. LXIrI 
$\operatorname{dim}(\Pi \backslash X)=\operatorname{Icd} X$. For each $i$ there exists an open subset $\widetilde{U}_{i}$ with $\widetilde{U}_{i} \cap X=U_{i}$. Then, $\cap\left\{\widetilde{U}_{i} \mid i=1,2, \ldots\right\}$ is a complete extension of $A$ and

$$
\operatorname{Icd} A \leqslant \operatorname{dim}\left(\cap\left\{\widetilde{U}_{i} \mid i=1,2, \ldots\right\} \backslash X\right) \leqslant \operatorname{dim}(I \backslash X)=\operatorname{Icd} X .
$$

The converse of this theorem holds for Icd $X=-1$ (theorem of Alexandrov-Hausdorff, 2.3). However, this converse does not hold in general as the following example shows.

EXAMPLE. In the notation of the example in 2.4, let $X$ be the disjoint union of a copy of $Q \times I^{m}$ and a copy of $I^{n}$. As is easily seen, $\operatorname{Icd} X=m$ (cf. the next theorem). Assume $m \geqslant n-1$ and $n \geqslant 1 . Q \times I^{n-1}$ can be regarded as a subset of the copy of $I^{n}$. Then, Icd $Q \times I^{n-1}=n-1 \leqslant m$. However, $Q \times I^{n-1}$ is not a $G_{\delta}$-subset of $X$. Indeed, if $Q \times I^{n-1}$ is a $G_{\delta}$-subset of $X$, then it is a $G_{\delta}$-subset of $I^{n}$ which implies $\operatorname{Icd}\left(Q \times I^{n-1}\right)=-1$. This contradicts the assumption $n \geqslant 1$.

4.5. Next, we discuss the analogy and the "dual" of the following sum theorem of dimension theory: If $X=A \cup B$, then $\operatorname{dim} X \leqslant \operatorname{dim} A+$ $+\operatorname{dim} B+1$ ([7], p. 19).

THeOREM. If $A$ and $B$ are subsets of $X$, then

$$
\begin{aligned}
& \operatorname{Icd}(A \cup B) \leqslant \operatorname{Icd} A+\operatorname{Icd} B+1, \\
& \operatorname{Icd}(A \cap B) \leqslant \operatorname{Icd} A+\operatorname{Icd} B+1 .
\end{aligned}
$$

Corollary. The Icd of a space cannot be increased by the adjunction of a complete space. The Icd of a subset of a space $X$ cannot be increased by the taking of the intersection with a complete subset of $X$.

Proof. Let $\widetilde{X}$ be a complete extension of $X$. Let $\widetilde{A}$ and $\widetilde{B}$ be $G_{\delta}$-subsets of $\widetilde{X}$ which contain $A$ and $B$ respectively such that

$$
\operatorname{dim}(\widetilde{A} \backslash A)=\operatorname{Icd} A \quad \text { and } \quad \operatorname{dim}(\widetilde{B} \backslash B)=\operatorname{Icd} B
$$

(main theorem and 2.3). $\widetilde{A} \cup \widetilde{B}$ is a complete space and

Similarly,

$$
\begin{aligned}
\operatorname{Icd}(A \cup B) & \leqslant \operatorname{dim}((\widetilde{A} \cup \widetilde{B}) \backslash(A \cup B)) \leqslant \operatorname{dim}((\widetilde{A} \backslash A) \cup(\widetilde{B} \backslash B)) \\
& \leqslant \operatorname{dim}(\widetilde{A} \backslash A)+\operatorname{dim}(\widetilde{B} \backslash B)+1=\operatorname{Icd} A+\operatorname{Icd} B+1 .
\end{aligned}
$$

$$
\begin{aligned}
\operatorname{Icd}(A \cap B) & \leqslant \operatorname{dim}[(\widetilde{A} \cap \widetilde{B}) \backslash(A \cap B)] \leqslant \operatorname{dim}((\widetilde{A} \backslash A) \cup(\widetilde{B} \backslash B)) \\
& \leqslant \operatorname{Icd} A+\operatorname{Icd} B+1 .
\end{aligned}
$$

4.6. The second sum theorem of dimension theory states 'that if $\left\{F_{y} \mid \gamma \in I\right\}$ is a locally countable closed covering of a space $X$ with $\operatorname{dim} F_{\gamma} \leqslant n$ for each $\gamma \in \Gamma$, then $\operatorname{dim} X \leqslant n([7], p .17)$. The analogy of this theorem is obviously false. The space $Q$ of the rationals is the countable union of singletons which obviously are complete. However, $\operatorname{Icd} Q=0$ as is easily seen. We have the following sum theorem.

THEOREM. Let $\left\{F_{\gamma} \mid \gamma \in \Gamma\right\}$ be a locally finite cover of a space $X$ such that $F_{\gamma}$ is open or closed and $\operatorname{Icd} F_{y} \leqslant n$ for each $\gamma \in \Gamma$. Then $\operatorname{Icd} X \leqslant n$.

Proof. Let $\tilde{X}$ be a complete extension of $X$. In view of lemma 4.3 we may assume that $\left\{F_{\gamma} \mid \gamma \in \Gamma\right\}$ is a locally finite collection of subsets of $\tilde{X}$. For each $\gamma \in \Gamma$, let $G_{\gamma}$ be a $G_{\delta}$-subset of $\widetilde{X}$ such that

(1) $F_{\gamma}=G_{\gamma} \cap X$

(2) $G_{\gamma} \subset \bar{F}_{\gamma}$,

(3) $\operatorname{dim}\left(G_{\gamma} \backslash F_{\gamma}\right)=\operatorname{Icd} F_{\gamma}$, and

(4) if $F_{\gamma}$ is open, then $G_{\gamma} \subset U_{\gamma}$, where $U_{\gamma}$ is an open subset of $\widetilde{X}$ such that $\bar{U}_{\gamma} \cap \bar{X}=F_{\gamma}$.

The existence of such a $G_{\delta}$-set $G_{\gamma}$ is proved as follows. By 2.3 , there exists a $G_{\delta}$-set $E_{\gamma}$ in $\widetilde{X}$ such that $F_{\gamma} \subset E_{\gamma}$ and $\operatorname{dim}\left(E_{\gamma} \backslash F_{\gamma}\right)=\operatorname{Icd} F_{\gamma}$. If $F_{\gamma}$ is closed in $X$, one takes the intersection of $E_{\gamma}$ and the $G_{\delta}$ - subset $\bar{F}_{\gamma}$ of $\tilde{X}$. This set satisfies conditions (1), (2), and (3). If $F_{\gamma}$ is open, then $E_{\gamma} \cap U_{\gamma}$ is the required $G_{\delta}$-subset $G_{\gamma}$. Since $\left\{\bar{F}_{y} \mid \gamma \in \Gamma\right\}$ is a locally finite collection of subsets of $\widetilde{X}$, by (2) and lemma 4.2 it follows that $G=\left\{G_{\gamma} \mid \gamma \in \Gamma\right\}$ is a complete extension of $X$.

If $F_{\gamma}$ is closed in $X$, then $H_{\gamma}=\bar{F}_{\gamma} \backslash G_{\gamma}$ is an $F_{\sigma}$-subset of $\widetilde{X}$, since $\bar{F}_{\gamma}$ is closed and $\bar{F}_{\gamma} \backslash G_{\gamma}$ is an $F_{\sigma}$ - subset of $\bar{F}_{\gamma}$. If $F_{\gamma}$ is open in $X$, then $U_{\gamma} \backslash G_{\gamma}$ $=H_{\gamma}$ is an $F_{\sigma}$-subset of $\widetilde{X}$, since $H_{\gamma}$ is the intersection of $U_{\gamma}$ and $\widetilde{X} \backslash G_{\gamma}$, both $U_{\gamma}$ and $\widetilde{X} \backslash G_{\gamma}$ being $F_{\sigma}$-subsets of $\widetilde{X}$. Each $H_{\nu}$ is contained in $\tilde{X} \backslash X$. Since $H_{\gamma} \subset \bar{F}_{\gamma}$ for each $\gamma \in \Gamma,\left\{H_{\gamma} \mid \gamma \in \Gamma\right\}$ is locally finite. By lemma 3.3 we have $\bigcup\left\{H_{\gamma} \mid \gamma \in \Gamma\right\}$ is an $F_{\sigma}$-subset of $\widetilde{X}$. We delete this $F_{\sigma}$-subset from $G$ and obtain a complete extension $G_{1}$ of $X$. If $F_{\gamma}$ is closed in $X$, then

$$
\bar{F}_{\gamma} \cap\left(G_{1} \backslash X\right)=G_{\gamma} \cap\left(G_{1} \backslash X\right) .
$$

If $F_{\gamma}$ is open in $X$, then

$$
U_{\gamma} \cap\left(G_{1} \backslash X\right)=G_{\gamma} \cap\left(G_{1} \backslash X\right) .
$$

Then we have

$$
G_{1} \backslash X=\bigcup\left\{G_{\gamma} \cap\left(G_{1} \backslash X\right) \mid \gamma \in \Gamma\right\},
$$

$\left\{G_{\gamma} \cap\left(G_{1} \backslash X\right) \mid \gamma \in \Gamma\right\}$ is a locally finite collection of subsets of $G_{1} \backslash X$, $G_{y} \cap\left(G_{1} \backslash X\right)$ is open or closed in $G_{1}$ for each $\gamma \in \Gamma$ by $(*)$, and $\operatorname{dim}\left(G_{\gamma} \cap\left(G_{1} \backslash X\right)\right) \leqslant n$ by (3). By the sum theorem of dimension theory it now follows that $\operatorname{dim}\left(G_{1} \backslash X\right) \leqslant n$ (observe that in a metric space each open set is an $F_{\sigma}$-subset). By the main theorem $\operatorname{Icd} X \leqslant n$.

The following example may illustrate the foregoing sum theorems. 
Example. The unit interval $I$ is the union of two $G_{\delta}$-subsets $Z_{1}$ and $Z_{2}$ each of which is zero-dimensional (by [8], p. 19 and p. 32). $Z_{1} \times Q$ and $Z_{2} \times Q$ will be regarded as subsets of $I \times Q$ (as for notation see 2.4). Then $I \times Q=Z_{1} \times Q \cup Z_{2} \times Q$ and $\operatorname{Icd} Z_{1} \times Q=\operatorname{Icd} Z_{2} \times Q=0$, as is easily seen. By Theorem 4.5 we have Icd $I \times Q \leqslant 1$. Actually, in $2.4 \operatorname{Icd} I \times Q=1$ has been proved. This example shows that the condition " $F_{\gamma}$ is open or closed" cannot be replaced by " $F_{\gamma}$ is a $G_{\delta}$-subset".

4.7. It is an open problem whether or not the following decomposition theorem holds for completeness degree (cf. [7], p. 19). Let $n \geqslant 0$. Icd $X \leqslant n$ if and only if $X=A_{1} \cup \ldots \cup A_{n+1}$ for some $n+1$ subsets $A_{i}$ with Icd. $A_{i} \leqslant 0, i=1, \ldots, n+1$. The "if"-part is a consequence of theorem 4.5, bnt the construction of a decomposition is an open problem.

Observe that in dimension theory the decomposition theorem is a consequence of the sum theorem. If the decomposition theorem holds true for completeness degree, this might lead to a proof of the independence of the decomposition theorem from the sum theorem in dimension theory. The decomposition theorem holds true in case $\operatorname{Icd} X=\operatorname{dim} X$. Indeed, if $\operatorname{Icd} X=\operatorname{dim} X \leqslant n$, then $X$ can be decomposed in $n+1$ zerodimensional subsets $A_{1}, \ldots, A_{n+1}$ each of which has Ied $=0$ (otherwise $\operatorname{Icd} A_{i} \leqslant \operatorname{dim} A_{i}=0$ and theorem 4.5 lead to a contradiction). Thus, for example, $Q \times I^{n}$ can be decomposed into $n+1$ subsets $A_{1}, \ldots, A_{n+1}$ such that $\operatorname{Ied} A_{i}=0, i=1, \ldots, n+1$. lished.

However, the "dual" of the decomposition theorem is easily estab-

StRUCTURe Theoren. Icd $X \leqslant n$ if and only if, in some $X$-containing space $Y, X$ is the intersection of $n+1$ sets $X_{i}$ such that $\operatorname{Icd} X_{i} \leqslant 0$, $i=1, \ldots, n+1$.

Proof. The "if"-part follows from 4.5.

"only if"-part. Let $Y$ be a complete extension of $X$ such that $\operatorname{dim}(Y \backslash X) \leqslant n$. Decompose $Y \backslash X$ into $n+1$ zero-dimensional subsetis $A_{1}, \ldots, A_{n+1}$. Put $X_{i}=Y \backslash A_{i}$ and by the main theorem the "only if"-part follows.

4.8. As shown in 2.4, there is no product theorem for completeness degree. But we do have:

THEOREM. If $B$ is complete, then

$$
\operatorname{Icd}(A \times B) \leqslant \operatorname{Ied} A+\operatorname{dim} B .
$$

Proof. If $\widetilde{A}$ is a complete extension of $A$, then $\widetilde{A} \times B$ is a complete extension of $A \times B$. The theorem now follows from the product theorem of dimension theory ([7], p. 20) and the main theorem.
5. Some characterizations of Icd. In this section we give two characterizations of Icd which are motivated by well-known theorems of dimension theors. The first characterization is prored by modifying the method of 3.4 .

5.1. Theoren. Let $n \geqslant 0$. $\operatorname{Icd} X \leqslant n$ if and only if there exists $\sigma$-locally finite open base $\mathcal{B}$ for $X$ such that $\operatorname{Ied} B(V) \leqslant n-1$ for every $V \in \mathcal{B}$.

This theorem should be compared with Theorem II 2 in [7], p. 32 .

Proof. "only if"-part. In $X$ there exists a $\sigma$-locally finite collection of subsets $\left\{U_{\gamma} \mid \gamma \in \Gamma\right\}$ and a collection of closed subsets $\left\{F_{y} \mid \gamma \in \Gamma\right\}$ such that

1) $F_{\gamma} \subset U_{\gamma}$ for each $\gamma \in \Gamma$, and

2) if $V_{\gamma}, \gamma \in \Gamma$, is an open subset such that $F_{\gamma} \subset V_{\gamma} \subset U_{\gamma}$, then $\left\{V_{\gamma} \mid \gamma \in \Gamma\right\}$ is a $\sigma$-locally finite base (see 3.4, B). If Ied $X \leqslant n$, by lemma 3.2 for each $\gamma \in \Gamma$ there exists an open set $W_{\gamma}$ such that $F_{\gamma} \subset W_{\gamma} \subset U_{\gamma}$ and Icd $B\left(W_{\gamma}\right) \leqslant n-1$. Obviously, $\left\{W_{\gamma} \mid \gamma \in I\right\}$ satisfies all conditions of the theorem.

"if"-part. Let $\Re=\left\{V_{\gamma} \mid \gamma \in T\right\}$ be a $\sigma$-locally finite base for $X$ such that $\operatorname{Ied} B\left(V_{\gamma}\right) \leqslant n-1$ for each $\gamma \in \Gamma$. Let $\widetilde{X}$ be a complete extension of $X$. For each $\nabla_{\gamma} \in \Re$, let $\widetilde{V}_{\gamma}$ be an open subset of $\widetilde{X}$ such that $\widetilde{V}_{\gamma} \cap X=V_{\gamma}$. Now (in some fixed metric of $\widetilde{X}$ ), the diameter of $\widetilde{V}_{\gamma}$ is equal to the diameter of $V_{\gamma}$ for each $\gamma \in T$, since $X$ is a dense subset of $\widetilde{X}$. Let $\widetilde{U}_{i}$ denote the union of all elements $\widetilde{V}_{y}$ which satisfy the condition diameter $\widetilde{V}_{\gamma}<1 / i$. Obviously, $\widetilde{U}_{i}$ is an open subset of $\widetilde{X}, i=1,2, \ldots$ Now, write $\mathfrak{B}=\bigcup\left\{\mathscr{B}_{i} \mid i=1,2, \ldots\right\}$ where each $\mathfrak{B}_{i}$ is locally finite. By lemma 4.3 there exists an open subset $\widetilde{W}_{i}$ of $\widetilde{X}$ such that $X \subset \widetilde{W}_{i}$ and $\mathcal{B}_{i}$ is a locally finite collection in the subspace $\widetilde{W}_{i} ; i=1,2, \ldots$

Then, $Y=\cap\left\{\widetilde{W}_{i} \cap \widetilde{U}_{i} \mid i=1,2, \ldots\right\}$ is a $G_{\delta}$-subset of $\tilde{X}$ which contains $X$ and for which the family $\{\tilde{V} \cap Y \mid V \in \mathscr{B}\}$ is a $\sigma$-locally finite base.

Now, the proof is completed as follows. For each $\gamma \in \Gamma$, the set $B_{X}\left(V_{\gamma}\right) \subset B_{Y}\left(\widetilde{V}_{\gamma} \cap Y\right)$. Since $\operatorname{Icd} B_{X}\left(V_{\gamma}\right) \leqslant n-1$, by the main theorem and lemma 2.3 there exists a complete set $C_{y}$ such that $B_{X}\left(V_{y}\right) \subset C_{y} \subset B_{Y}\left(\widetilde{V}_{y} \cap Y\right)$ and $\operatorname{dim} C_{\gamma} \backslash B_{X}\left(V_{y}\right) \leqslant n-1$. As in $3.4 \mathrm{E}$ and $\mathrm{F}, \bigcup\left\{B_{Y}\left(\widetilde{V}_{\gamma} \cap Y\right) \backslash C_{y} \mid \gamma \in \Gamma\right\}$ is deleted from $Y$ and the remaining space $Y_{1}$ satisfies $\operatorname{dim}\left(Y_{1} \backslash X\right) \leqslant n$. Consequently, by the main theorem $\operatorname{Icd} X \leqslant n$.

5.2. In dimension theory the notions "order of a covering" and "order of the system of boundaries of a base" are very important. Keeping this in mind we can except the usefullness of a condition of the form: the intersection of any $n+1$ members of a collection is complete.

We have the following theorem (cf. [7], p. 32). 
THEOREM. Icd $X \leqslant n$ if and only if there is a $\sigma$-locally finite base $\left\{V_{y} \mid \gamma \in \Gamma\right\}$ for $X$ such that $B\left(\nabla_{\gamma_{1}}\right) \cap \ldots \cap B\left(\nabla_{\gamma_{n+1}}\right)$ is complete for each set of $n+1$ different indices.

Proof. "if"-part. The case $n=-1$ is obvious. For $n=0$, it is a rewording of the "if" -part of the preceeding theorem. In view of the preceeding theorem if suffices to show that for each $\gamma \in T$ we have $\operatorname{Ied} B\left(V_{\gamma}\right) \leqslant n-1$. Let $n \geqslant 1 . \quad\left\{B\left(V_{\gamma}\right) \cap V_{\beta} \mid \beta \neq \gamma, \beta \in \Gamma\right\}$ is a $\sigma$-locally finite base for $B\left(V_{\gamma}\right)$ and $B_{B\left(V_{\gamma}\right)}\left(V_{\beta}\right) \subset B\left(V_{\beta}\right)$. Then, for each set of $n+1$ different indices $\gamma, \gamma_{1}, \ldots, \gamma_{n}$ we have

$$
B_{B\left(V_{\gamma}\right)}\left(V_{\gamma_{1}}\right) \cap \ldots \cap B_{B\left(V_{\gamma}\right)}\left(V_{\gamma_{n}}\right) \subset B\left(V_{\gamma}\right) \cap B\left(V_{\gamma_{1}}\right) \cap \ldots \cap B\left(V_{\gamma_{n}}\right)
$$

and the theorem follows by induction.

"only if"-part. Let $Y$ be a complete extension of $X$ such that $\operatorname{dim}(T \backslash X) \leqslant n$. As in $3.4 \mathrm{~B}$ let $\left\{U_{y} \mid \gamma \in \Gamma\right\}$ be a $\sigma$-locally finite open base for $Y$ and $\left\{F_{\gamma} \mid \gamma \in \Gamma\right\}$ a collection of closed sets such that $F_{\nu} \subset U_{\nu}$ and if $\nabla_{\gamma}, \gamma \in \Gamma$, is an open subset with $F_{\gamma} \subset V_{\gamma} \subset U_{\nu}$, then $\left\{\nabla_{\gamma} \mid \gamma \in \Gamma\right\}$ is a $\sigma$-locally finite base for $Y$. Now, for each $\gamma \in \Gamma$ there is an open set $U_{\gamma}$ and a closed set $F_{\gamma}^{\prime}$ such that $F_{\gamma} \subset$ interior $F_{\gamma}^{\prime} \subset F_{\gamma}^{\prime} \subset U_{\gamma}^{\prime} \subset \bar{U}_{\gamma}^{\prime} \subset U_{\gamma}$. By [7], pp. 25-26 in the subspace $\Pi \backslash X$ there exist open subsets $W_{\gamma}, \gamma \in \Gamma$, such that

and

$$
F_{\gamma}^{\prime} \cap(Y \backslash X) \subset W_{\gamma} \subset \bar{W}_{\gamma} \cap(Y \backslash X) \subset(Y \backslash X) \backslash U_{\gamma}^{\prime}
$$

$$
\bigcap\left\{\left(\bar{W}_{\gamma_{i}} \backslash W_{\gamma_{i}}\right) \cap(Y \backslash X) \mid i=1, \ldots, n+1\right\}=\emptyset
$$

for each set of $n+1$ different indices $\gamma_{1}, \ldots, \gamma_{n+1}$. For each $\gamma$ neither of the disjoint sets $F_{\gamma} \cup W_{\gamma}$ and $\left(Y \backslash U_{\gamma}\right) \cup\left((Y \backslash X) \backslash \bar{W}_{\gamma}\right)$ contains a cluster point of the other. By lemma 2.2 there exists an open set $V_{\gamma}$ such that $F_{\gamma} \subset \nabla_{\gamma} \subset \bar{V}_{\gamma} \subset \sigma_{\gamma}$ and $\left(\bar{V}_{\gamma} \backslash \nabla_{\gamma}\right) \cap(Y \backslash X) \subset\left(\bar{W}_{\gamma} \backslash W_{\gamma}\right) \cap(Y \backslash X), \gamma \in \Gamma$. It follows that $\cap\left\{\left(\bar{V}_{\gamma_{i}} \backslash V_{\gamma_{i}}\right) \cap(\bar{X} \backslash X) \mid i=1, \ldots, n+1\right\}=\varnothing$ for each set of $n+1$ different indices. Obviously,

$$
B_{X}\left(V_{\gamma_{1}}\right) \cap \ldots \cap B_{X}\left(V_{\gamma_{n+1}}\right) \subset\left(\bar{V}_{\gamma_{1}} \backslash V_{\gamma_{1}}\right) \cap \ldots \cap\left(\bar{V}_{\gamma_{n+1}} \backslash V_{\gamma_{n+1}}\right) .
$$

The last set is complete and it is contained in $X$ in the case where $\gamma_{1}, \ldots, \gamma_{n+1}$ are $n+1$ different indices. Now, the theorem easily follows.

\section{Small inductive completeness degree.}

6.1. By replacing the empty set in the definition of small inductive dimension ([7], p. 9) we get

Definition. A space $X$ has small inductive completeness degree -1 , ic $X=-1$, if $X$ is complete. If for every neighbourhood $U(p)$ of every point $p$ of $X$ there exists an open neighbourhood $V$ such that $p \in V \subset U(p)$ and icd $B(V) \leqslant n-1$, then $X$ has small inductive completeness degree $\leqslant n$, icd $X \leqslant n$. icd $X=n$ if icd $X \leqslant n$ and icd $X \leqslant n-1$. ied $X=\infty$ if $\operatorname{ied} X \$ n$ for each $n$.

Obviously, we have the inequality

$$
\operatorname{icd} X \leqslant \operatorname{Ied} X \text {. }
$$

We have

6.2. THEOREM. If $X$ is a separable metric space, then $\operatorname{ied} X=\operatorname{Icd} X$.

Proof. The proof is a copy of the proof of ind $X=\operatorname{Ind} X$ for separable spaces in [7], p. 90. We only have to replace ind and Ind by icd and Icd respectively. icd $X \leqslant \operatorname{Icd} X$ as observed above. Inductively on $\operatorname{icd} X$, we show that $\operatorname{icd} X \geqslant \operatorname{Icd} X$. If icd $X=-1$, this inequality is obvious. Suppose that icd $X=\operatorname{Icd} X$ has been proved correct for each space $X$ with $\operatorname{icd} X \leqslant n-1$. Suppose $\operatorname{icd} X=n$. Let $\left\{U_{i} \mid i=1,2, \ldots\right\}$ be a countable open base. If $\bar{U}_{i} \subset U_{j}$ and if there exists a $W$ such that $\bar{U}_{i} \subset W \subset U_{j}, \operatorname{icd} B(W) \leqslant n-1$, then we choose a fixed $W$ with this property and denote it by $W_{i j}$. Otherwise $W_{i j}=\varnothing$. If follows from $\operatorname{Icd} X=n$, that $\left\{W_{i j} \mid i, j=1,2 \ldots\right\}$ is an open base with $\operatorname{ied} B\left(W_{i j}\right)$ $\leqslant n-1$. By the induction hypothesis $\operatorname{Icd} B\left(W_{i j}\right) \leqslant n-1$. By theorem 5.1 we have $\operatorname{Icd} X \leqslant n$.

6.3. Due to this theorem all theorems, which are proved for Icd, hold for icd in the separable case. However, observe that several theorems can be proved directly, i.e. by induction and without using the main theorem. For example; if $A$ is a $G_{\delta}$-subset of $X$, then $\operatorname{icd} A \leqslant \operatorname{ied} X$.

$$
\operatorname{icd}(A \cup B) \leqslant \operatorname{icd} A+\operatorname{icd} B+1 \text {. }
$$

It is an open problem whether $\operatorname{icd} X=\operatorname{Ied} X$ in general.

\section{Covering completeness degree.}

7.1. Definitions. A border cover of a space $X$ is a family of open sets $\left\{U_{\gamma} \mid \gamma \in \Gamma\right\}$ such that $X \backslash \bigcup\left\{U_{\gamma} \mid \gamma \in \Gamma\right\}$ is complete. The order of a border cover $\mathcal{U}$ at $p$ is the number of members of $U$ which contain $p$ (of course, we allow order $+\infty$ ). The order of a border cover $น$ will be the supremum of the orders of $U$ at the points of $X$. A border cover $\left\{U_{\gamma} \mid \gamma \in T\right\}$ is a refinement of $\left\{V_{\delta} \mid \delta \in \Delta\right\}$ if for each $\gamma$ there is a $\delta$ such that $U_{\gamma} \subset V_{\delta}$.

7.2. Definition. If for any border cover $U$ of $X$ there exists a border cover $v$ such that $v$ refines $\mathcal{U}$ and order of $v \leqslant n+1$, then $X$ has covering completeness degree $\leqslant n$, $\operatorname{cocd} X \leqslant n$. If $\operatorname{cocd} X \leqslant n$ and $\operatorname{cocd} X \$ n-1$, then $\operatorname{cocd} X=n$. If $\operatorname{cocd} X \$ n$ for each $n$, then $\operatorname{cocd} X=\infty$.

Though cocd is quite different from Ied in the way it is defined, we have that cocd and Icd coincide. 
7.3. THEOREM. $\operatorname{coc} d X=\operatorname{Icd} X$.

Proof. $\operatorname{cocd} X \leqslant \operatorname{Icd} X$ : Let $Y$ be a complete extension of $X$ such that $\operatorname{dim} Y \backslash X=\operatorname{Ied} X=n$. Let $\left\{U_{\gamma} \mid \gamma \in \Gamma\right\}$ be a border cover of $X$. For each $\gamma$ an open subset $\widetilde{U}_{\gamma}$ of $Y$ is selected such that $\widetilde{U}_{\gamma} \cap X=U_{\gamma}$. Let $Y_{1}=X \cup\left\{\tilde{U}_{\gamma} \mid \gamma \in \Gamma\right\}$. Then $Y_{1}$ is a complete extension of $X$ such that $\operatorname{dim}\left(Y_{1} \backslash X\right)=n$ (the completeness of $Y_{1}$ follows from the fact that $\mathbb{Y}_{1}=\left(X \backslash \bigcup\left\{U_{\gamma} \mid \gamma \in \Gamma\right\}\right) \cup\left(\bigcup\left\{\widetilde{U}_{\gamma} \mid \gamma \in T\right\}\right)$, both summands being complete). Since $\operatorname{dim}\left(\bar{Y}_{1} \backslash X\right)=n$, the family $\left\{\widetilde{U}_{\nu} \cap\left(Y_{1} \backslash X\right) \mid \gamma \in \Gamma\right\}$ has an open refinement $\left\{V_{\delta} \mid \delta \in \Delta\right\}$ of order $\leqslant n+1 \cdot([7]$, p. 23). By [6], p. 122 it follows that in $Y_{1}$ there exists an open collection $\left\{W_{\delta} \mid \delta \in \Delta\right\}$ such that $W_{\delta} \cap\left(Y_{1} \backslash X\right)=\nabla_{\delta}$ and $V_{\delta_{1}} \cap \ldots \cap V_{\delta_{k}}=\emptyset$ implies $W_{\delta_{1}} \cap \ldots \cap W_{\delta k}=\emptyset$, $\delta_{1}, \ldots, \delta_{k} \in \Delta$. So, the order of $\left\{W_{\delta} \mid \delta \in \Delta\right\}$ does not exceed $n+1$. Finally, for each $\delta$ there exists a $\gamma$ such that $V_{\delta} \subset \widetilde{U}_{\gamma} \cap(X \backslash X)$. Let $O_{\delta}=W_{\delta} \cap \widetilde{U}_{\nu}$. Then $\left\{0_{\delta} \mid \delta \epsilon \Delta\right\}$ is a border cover of $X$ of order $n+1$ which refines $\left\{U_{\gamma} \mid \gamma \in \Gamma\right\}$. Hence $\operatorname{coc} d X \leqslant n$.

Icd $X \leqslant \operatorname{cocd} X$ : Let $Y$ be an arbitrary complete extension of $X$ and let $\varrho$ denote a metric of $Y$. Let $\mathcal{U}_{1}=\left\{S_{1}(x) \mid x \in X \backslash X\right\}$, where $S_{1}(x)$ $=\{y \in Y \mid \varrho(x, y)<1\}$. Let $\mathcal{U}_{1}^{\prime}=\left\{U \cap X \mid \quad U \in \mathcal{U}_{1}\right\}$ and let $\vartheta_{1}$ be a border cover of $X$ which is a refinement of $\mathcal{H}_{1}^{\prime}$ and has order $\leqslant \operatorname{cocd} X+1=n+1$. Then, for each $V \in \mho_{1}$ an open subset $W$ of $Y$ is selected such that $W \cap X$ $=\nabla$. The collection of all $W$, which are obtained in this way, is denoted by $w_{1}$. Since $X$ is dense in $Y$, we have order $w_{1} \leqslant n+1$. Suppose we have defined $\mathfrak{w}_{1}, \ldots, \mathfrak{w}_{k-1}$. Let $\mathcal{U}_{k}=\left\{S_{1 / k}(x) \mid x \in \prod \backslash X\right\}$ and let $\mathcal{U}_{k}^{\prime}=\{U \cap X \mid$ $\left.U \epsilon \mathcal{U}_{k}\right\}$. Let $\mho_{k}$ be a border cover of $X$ which is a refinement of both $\mathcal{W}_{k}^{\prime}$ and $V_{k-1}$ and which has order $\leqslant n+1$. Then, for each $V \epsilon V_{k}$ an open subset $W$ of $Y$ is selected such that $W \cap X=V$. This can be done in such a way that $W$ is contained in some member of $W_{k-1}$ since $\mho_{k}$ is a refinement of $\vartheta_{k-1}$. $W_{k}$ is the collection of all $W$ which are obtained in this way. For each $k$, the set $Y_{k}=X \cup\left\{\bigcup\left\{W \mid W \in W_{k}\right\}\right\}$ is complete. It follows that $Y_{k}$ is a $G_{\delta}$ - subset of $Y$. Consequently, $Z=\cap\left\{Y_{k} \mid k=1,2, \ldots\right\}$ is a complete extension of $X$. We will $\operatorname{show} \operatorname{dim}(Z \backslash X) \leqslant n$. Let $W_{k}^{\prime}=\{W \cap$ $\left.\cap(Z \backslash X) \mid W \in W_{k}\right\}$. Then $\left\{W_{k}^{\prime} \mid k=1,2, \ldots\right\}$ is a sequence of open coverings of $\mathbb{Z} \backslash X$ such that

1) $w_{k+1}^{\prime}$ is a refinement of. $w_{k}^{\prime}, k=1,2, \ldots$,

2) order $w_{k}^{\prime} \leqslant n+1, k=1,2, \ldots$,

3) mesh $w_{k}^{\prime} \rightarrow 0$ as $k \rightarrow \infty$.

By [7], p. 126 it follows that $\operatorname{dim}(Z \backslash X) \leqslant n$. Hence Icd $X \leqslant n$.

\section{References}

[1] J. M. Aarts, Dimension and deficiency in general topology. Thesis, Amsterdam 1966.

[2] J. de Groot, Topologisehe Studien. Thesis, Groningen 1942.
[3] J. de Groot, Seminar on compactitication and dimension in metric spaces (mimeographed report)

[4] - and T. Nishiura, Inductive compactness as a generalization of semicompactness, Fund. Math. 58 (1966), pp. 201-218.

[5] J. L. Kelley, General topology, Princeton (N. J.) 1955.

[6] C. Kuratowski, Topologie I (4-th ed.), Warszawa 1958.

[7] J. Nagata, Modern dimension theory, Groningen 1965.

[8] T. Nishiura, Semicompact spaces and dimension, Proc. Amer. Math. Soc. 12 (1961), pp. 922-924.

[9] - Inductive invariants and dimension theory, Fund. Math. 59 (1966), pp. 243-262.

MASSACHUSETTS INSTITUTE OF TECHNOLOGY

Cambriase, Massachusetts

Reçu par la Rédaction le 2\%. 4. $196 \%$ 